\title{
Molecular Epidemiology of Clostridium difficile Infection in a Large Teaching Hospital in Thailand
}

\author{
Popchai Ngamskulrungroj ${ }^{1}$, Sittinee Sanmee ${ }^{1}$, Papanin Pusathit ${ }^{2}$, Pipat Piewngam ${ }^{1}$, \\ Briony Elliott' ${ }^{2}$, Thomas V. Riley ${ }^{2,3}$, Pattarachai Kiratisin ${ }^{1 *}$ \\ 1 Department of Microbiology, Faculty of Medicine, Siriraj Hospital, Mahidol University, Bangkok, Thailand, \\ 2 School of Pathology \& Laboratory Medicine, The University of Western Australia, Crawley, Australia, \\ 3 PathWest Laboratory Medicine WA, Queen Elizabeth II Medical Centre, Nedlands, Australia \\ * pattarachai.kir@mahidol.ac.th
}

\section{OPEnACCESS}

Citation: Ngamskulrungroj P, Sanmee S, Pusathit P, Piewngam P, Elliott B, Riley TV, et al. (2015) Molecular Epidemiology of Clostridium difficile Infection in a Large Teaching Hospital in Thailand. PLOS ONE 10(5): e0127026. doi:10.1371/journal. pone. 0127026

Academic Editor: Gayatri Vedantam, University of Arizona, UNITED STATES

Received: January 3, 2015

Accepted: April 10, 2015

Published: May 22, 2015

Copyright: @ 2015 Ngamskulrungroj et al. This is an open access article distributed under the terms of the Creative Commons Attribution License, which permits unrestricted use, distribution, and reproduction in any medium, provided the original author and source are credited.

Data Availability Statement: GenBank accession numbers for new sequences are KJ130417-446.

Funding: This work was supported by a research grant (to PK) from the Faculty of Medicine Siriraj Hospital, Mahidol University (Bangkok, Thailand) (grant no. R015333009). PN and PK were also supported by a 'Chalermphrakiat' grant, Faculty of Medicine Siriraj Hospital, during this study. The funders had no role in study design, data collection and analysis, decision to publish, or preparation of the manuscript.

\section{Abstract}

Clostridium difficile infection (CDI) is a leading cause of healthcare-associated morbidity and mortality worldwide. In Thailand, CDI exhibits low recurrence and mortality and its molecular epidemiology is unknown. CDI surveillance was conducted in a tertiary facility (Siriraj Hospital, Bangkok). A total of 53 toxigenic $C$. difficile strains from Thai patients were analyzed by multi-locus sequence typing (MLST), PCR ribotyping, and pulse-field gel electrophoresis (PFGE). The mean age of the cohort was 64 years and $62.3 \%$ were female; $37.7 \%$ of patients were exposed to > two antibiotics prior to a diagnosis of CDI, with beta-lactams the most commonly used drug (56.3\%). Metronidazole was used most commonly $(77.5 \%$; success rate $83.9 \%$ ), and non-responders were treated with vancomycin (success rate $100 \%)$. None of the isolates carried binary toxin genes. Most isolates $(98.2-100 \%)$ were susceptible to metronidazole, vancomycin, tigecycline and daptomycin. There were 11 sequence types (STs), 13 ribotypes (RTs) and four PFGE types. Six previously identified STs (ST12, ST13, ST14, ST33, ST41 and ST45) and five novel STs unique to Thailand (ST66, ST67, ST68, ST69 and ST70) were identified. PCR RTs UK 017 (ST45) (45.3\%) and UK 014/020 (ST33) (24.5\%) were the most common. High concordance was observed between the MLST and ribotyping results $(p<0.001)$. C. difficile isolates from Thai patients were highly susceptible to standard antimicrobial agents. In conclusion, the five STs indicate the high genetic diversity and unique polymorphisms in Thailand. Moreover, the emergence of antimicrobial resistance to vancomycin warranted continuous surveillance to prevent further spread of the toxigenic $C$. difficile isolates.

\section{Introduction}

Clostridium difficile, an obligate anaerobic Gram-positive bacillus, is an important etiological agent of antibiotic-associated diarrhea (C. difficile-associated diarrhea; CDAD or Clostridium difficile infection; $\mathrm{CDI}$ ). The disease is largely considered a healthcare-associated infection, 
Competing Interests: The authors have declared that no competing interests exist. with symptoms ranging from mild diarrhea to severe pseudomembranous colitis, which can occur during antibiotic treatment or shortly thereafter [1]. Two large clostridial toxins (LCTs), one an enterotoxin (toxin A; TcdA), the other a cytotoxin (toxin B; TcdB), are produced by toxigenic strains of $C$. difficile. The toxins interfere with the activity of proteins important for regulating actin polymerization, thereby leading to the disruption of the cell's cytoskeleton [1]. Toxigenic strains capable of causing CDI produce both toxins; however, toxin A-negative strains are increasingly being reported [2-4].

An additional toxin discovered in 1997, the actin perturbing binary toxin (CDT), has been reported to be present in 4-12\% of toxigenic C. difficile [5]. This toxin consists of two independent components comprising a catalytic (CDTa) and binding (CDTb) domain. Although the contribution of $\mathrm{CDT}$ to the virulence of $C$. difficile remains controversial, its biological function and its genetic and immunological properties are reported to closely resemble those of Clostridium perfringens iota toxin [6]. Interestingly, studies have reported synergy between CDT and LCTs [7,8], and that the presence of CDT might be an indicator for recurrent CDI [9].

As the prevalence of CDI increases globally as a consequence of high levels of antibiotic use, several techniques for investigating its epidemiology have been developed. Pulse-field gel electrophoresis (PFGE), PCR ribotyping and restriction endonuclease analysis have been used widely as typing methods [10]. Because gel banding patterns can be difficult to compare across laboratories, two multi-locus sequence typing (MLST) methods were recently established $[11,12]$. Use of the established MLST scheme for C. difficile and the publicly available online database of seven C. difficile housekeeping genes comprising aroE (shikimate dehydrogenase), dutA (dUTP pyrophosphatase), gmk (guanylate kinase), groEL (60 kDa chaperonin), recA (recombinase), sodA (superoxide dismutase) and tpi triosephosphate isomerase) enabled an inter-laboratory comparison of up to 65 of the sequence types (STs) present in the Pasteur Institute database [11]. The other MLST method, based on a different set of seven housekeeping genes, namely, adk (adenylate kinase), atpA (ATP synthase subunit alpha), $d x r$ (1-deoxy-Dxylulose 5-phosphate reductoisomerase), sodA (superoxide dismutase), recA (recombinase A), $g l y A$ (serine hydroxymethyltransferase) and tpi (triose phosphate isomerase) had similar discriminatory power [12].

$\mathrm{CDI}$ is frequently reported to be associated with previous exposure of the bacterium to clindamycin, cephalosporins and fluoroquinolones. Treatment for CDI can include antibiotic discontinuation; however, this alone is not always effective and antibiotic treatment is necessary in moderate to severe cases [1]. Because the antibiotics recommended in standard treatment regimens are metronidazole and vancomycin [13], the susceptibilities of C. difficile strains to these agents have been investigated [14]. In most cases, a high degree of resistance was observed against antibiotics reported to be associated with CDI, and most strains were susceptible to the antibiotics recommended in the standard treatment regimen for this disease [14].

In Thailand, several studies have investigated the clinical and microbiological aspects of CDI [15-17]. In 1990, a study showed the prevalence of fecal toxin B was $52.5 \%$ among Thai diarrheal patients [15]. More recently, studies reported beta-lactam antibiotics to be the most commonly used drugs prior to the onset of CDAD [16]. Additionally, CDI was reported to have low recurrence and mortality rates [17]. However, at present, data regarding the molecular epidemiology and antibiotic susceptibility profile of Thai $C$. difficile isolates is limited. Thus, this study aimed to perform the first comprehensive epidemiological study of toxigenic C. difficile isolated from Thai patients. 


\section{Materials and Methods Isolates and growth media}

The 53 toxin-positive $C$. difficile strains used in this study were isolated from the stools of patients who were diagnosed with suspected CDI and had been admitted to Siriraj Hospital, Bangkok, Thailand, between 2006 and 2008. This study was approved by the Siriraj Institutional Review Board [approval no. Si414/2007]. The requirement for informed consents was waived as the study was a retrospective chart review. Patient records/information was anonymized and de-identified prior to analysis. Toxins in the isolates were detected with a qualitative immunochromatographic assay (Xpect C. difficile toxin A/B test; Thermo scientific, Lenexa, KS, USA), according to the manufacturer's instructions. All toxin-positive samples identified in this manner were confirmed by toxin gene detection (as described below). Only toxin gene-positive strains were included in subsequent studies. Duplicate specimens from the same patients were excluded. Species identification was done according to internationally agreed standard testing procedures, which includes $16 \mathrm{~S}$ ribosomal RNA sequence analysis [18]. Strains were maintained on blood agar plates at $37^{\circ} \mathrm{C}$ in anaerobic conditions and in $30 \%$ glycerol at $-80^{\circ} \mathrm{C}$ for long term storage.

\section{Antibiotic susceptibility tests}

Susceptibility of the toxigenic $C$. difficile strains to vancomycin, metronidazole, ciprofloxacin, moxifloxacin, linezolid, tigecycline and daptomycin was determined using an E-test as previously described [19]. The quality control strains for Gram-positive tests were Staphylococcal aureus ATCC 29213. Briefly, a suspension equivalent to a $0.5 \mathrm{McFarland}$ standard for each isolate was prepared in nutrient broth (Oxoid Ltd, Basingstoke, UK) and swabbed onto supplemented (hemin and vitamin K1) Brucella agar containing 5\% sheep blood (BBL Microbiology Systems, Cockeysville, MD). An E-test strip (AB Biodisk, Solna, Sweden) was applied to each plate and the plates were incubated at $37^{\circ} \mathrm{C}$ in anaerobic conditions for $48 \mathrm{~h}$. The results were interpreted using the epidemiological cutoff values recommended by the European Committee on Antimicrobial Susceptibility Testing (EUCAST, http://www.eucast.org/) [20].

\section{DNA extraction}

Genomic DNA was prepared from C. difficile grown on blood agar overnight at $37^{\circ} \mathrm{C}$ in anaerobic conditions. A full loop (approximately $10 \mu \mathrm{l}$ ) of cells was harvested and used for subsequent DNA extraction. High molecular weight DNA was extracted using a High Pure PCR Template Preparation Kit (Roche Applied Science, Indianapolis, IN) according to the manufacturer's instructions.

\section{Detection of $C$. difficile toxin genes}

$t c d A, t c d B, c d t A$ and $c d t B$ genes were detected in the isolates using a 5-plex PCR containing 12 primers specific to the four toxin genes and a control gene, 16S rDNA (S1 Table). The multiplex PCR assay was performed according to a previously published method [21]. Subsequently, the isolates were screened with an in-house PCR for the presence of toxin A $(t c d A)$ [22] and for changes in the $t c d A$ repetitive region [22].

\section{PCR ribotyping}

Ribotyping of the isolates based on the $16 \mathrm{~S}-23 \mathrm{~S}$ intergenic spacer region was done as previously described [23]. PCR amplifications were performed using the following primers: 5'-CTGGGG TGAAGTCGTAACAAGG-3' and 5'-GCGCCCTTTGTAGCTTGACC-3'. The PCR conditions 
comprised 35 cycles of denaturation at $94^{\circ} \mathrm{C}$ for $1 \mathrm{~min}$, annealing at $55^{\circ} \mathrm{C}$ for $1 \mathrm{~min}$ and extension at $72^{\circ} \mathrm{C}$ for $2 \mathrm{~min}$. Amplification products were concentrated and electrophoresis was performed on $3 \%$ agarose gels. The typing patterns generated were compared with those of a previously published library [10].

\section{PFGE}

PFGE was performed according to a previously published method [24]. Briefly, the isolates were grown in pre-reduced Schaedler's anaerobic broth at $37^{\circ} \mathrm{C}$ overnight in an anaerobic atmosphere. The bacterial cells were pelleted and lysed in $100 \mu \mathrm{l}$ of lysis buffer (10 mM Tris, 0.5 mM EDTA, $0.8 \% \mathrm{~N}$-lauryl sarcosine, $5 \mathrm{mg} / \mathrm{ml}$ lysozyme). Plugs containing the bacterial cells were made using $2 \%$ PFGE-grade agarose with a low melting point. The bacterial plugs were then incubated in the lysis buffer for $1 \mathrm{~h}$ at $37^{\circ} \mathrm{C}$ followed by incubation in $0.5 \mathrm{mM}$ EDTA, $1 \%$ $\mathrm{N}$-lauryl sarcosine, $10 \mathrm{mg} / \mathrm{ml}$ proteinase $\mathrm{K}$, overnight at $50^{\circ} \mathrm{C}$. The plugs were washed and digested with $\mathrm{SmaI}$ restriction enzyme $(20 \mathrm{U})$ for $5 \mathrm{~h}$ at $30^{\circ} \mathrm{C}$. The digested products were run on a $1 \%$ PFGE-grade agarose gel using a CHEF Mapper (Bio-Rad, UK) with previously described settings [24]. The gels were documented and analyzed with a Fingerprinting II program (BioRad). Dendrograms were constructed by the unweighted pair-group method with arithmetic mean clustering using the Dice correlation coefficient [25].

\section{MLST}

MLST was performed and analyzed according to the methodologies of previous publications [11]. Briefly, seven unlinked genetic loci, aroE (shikimate dehydrogenase), dutA (dUTP pyrophosphatase), gmk (guanylate kinase), groEL (60 kDa chaperonin), recA (recombinase), sodA (superoxide dismutase) and $t p i$ (triosephosphate isomerase) were amplified and sequenced. The sequences generated were manually edited using BioEdit 7.0.9.0, [25] and allele types (ATs) and STs for each locus were assigned according to the C. difficile MLST database at the Pasteur Institute (http://www.pasteur.fr/recherche/genopole/PF8/mlst/Cdifficile2.html) as previously described [12].

\section{Statistical analysis and correlation analysis}

The PASW Statistics 18 program (IBM, Armonk, NY) was used to calculate frequencies of demographic data, antibiotic susceptibility, ATs and STs. Correlation analysis was done using a Lambda method for nominal variables by the PASW Statistics 18 program. Correlation and significance was defined when Lambda value was close to 1 and p-value was less than or equal to 0.05 , respectively. Only MLST types with more than or equal to 3 members were subjected to the association analysis.

\section{Results}

\section{C. difficile strains and patient demography}

To obtain a complete overview of the epidemiology of pathogenic C. difficile, all strains isolated from 2006-2008 were tested for toxin production. Using immunochromatographic assays, a total of 53 C. difficile strains tested positive for toxin. The results of the multiplex PCR assay showed that these strains were also positive for $t c d A$ and $t c d B$; therefore, they were included in this study. The cases from which C. difficile was isolated were aged 1-97 years, with a mean age of 64 years. The majority of patients were female (62.3\%).

Analysis of the patient demography showed that $57.6 \%$ of the patients were being treated with antibiotics when CDI was diagnosed. We found that CDI can occur as late as 16 days 
post-antibiotic discontinuation (mean value of 2.52 days). As studies have shown that CDI can occur long after antibiotic exposure [1], here, we looked for a history of antibiotic use in the 2 months prior to diagnosis. During this period, patients with CDI were treated with zero to seven antibiotics, with $66.0 \%$ and $37.7 \%$ of them having used more than one and two antibiotics, respectively (S2 Table); 18.9\% of the patients had no history of antibiotic use. Of the 126 antibiotic administration events, beta-lactams were the most frequently used drug $(56.3 \%$, Table 1).

Metronidazole was the most common antimicrobial used for treatment $(77.5 \%$; 31 of 40 patients with available data) with a cure rate of $83.9 \%$ ( 26 of 31 patients with available data). Vancomycin was administered for 14 days in three cases where the patients failed to respond to metronidazole treatment; this resulted in a $100 \%$ cure rate (S2 Table). The other two patients died from complications; hence the efficacy of metronidazole could not be determined.

\section{Antibiotic susceptibility of $C$. difficile}

The minimum inhibitory concentration (MIC) of each antibiotic $\left(\mathrm{MIC}_{50}\right.$ and $\mathrm{MIC}_{90}$ ) is shown in Table 2. All C. difficile isolates were inhibited by a concentration of $\leq 0.5 \mu \mathrm{g} / \mathrm{mL}$ of metronidazole. All but two isolates were inhibited by a concentration of $\leq 0.75 \mu \mathrm{g} / \mathrm{mL}$ of vancomycin. Very high MICs $(>32 \mu \mathrm{g} / \mathrm{mL})$ were observed in all isolates tested with ciprofloxacin and in $43 \%$ of the isolates (23/53) tested with moxifloxacin. Most isolates exhibited low MICs $(<0.5 \mu \mathrm{g} / \mathrm{mL}$ ) against linezolid, tigecycline and daptomycin (Table 2). Only moxifloxacin showed a considerable number of resistant isolates (45.2\%) thus was subjected to correlation analysis. No correlation between the resistance with MLST, ribotype or PFGE type was observed ( $\mathrm{p}$-value $=0.818,0.808$ and 0.170 , respectively).

\section{Molecular detection of $C$. difficile toxin}

$t c d A$ and $t c d B$ genes were both present in all isolates, although 23 of them had a partial deletion in $t c d A(\mathrm{~A}-\mathrm{B}+$ ). These included all strains from ribotypes (RTs) UK 017 (Th2, Th3, Th4, Th6, Th7, Th8, Th10, Th11, Th12, Th14, Th19, Th24, Th27, Th34, Th38, Th39, Th41, Th43, Th44, Th47, Th49 and Th53) and QX370 (Th45) (S2 Table). No isolates contained binary toxin genes ( $c d t A$ and $c d t B)$.

\section{PFGE}

Four PFGE types were recognized with type 2 being the most common (43.4\%, Fig 1).

Table 1. Antibiotic exposure in the 2 month period preceding a diagnosis of CDI in patients.

\begin{tabular}{|c|c|}
\hline Antibiotic groups & Percent \\
\hline $\begin{array}{l}\text { Beta-lactams amoxicillin-clavulanic acid }(0.8 \%) \text {, cefdinir }(0.8 \%) \text {, cefditoren }(0.8 \%) \text {, cefipime } \\
(4 \%) \text {, cefotaxime }(1.6 \%) \text {, ceftazidime }(5.6 \%) \text {, ceftriaxone }(9.5 \%) \text {, cefuroxime }(0.8 \%) \text {, cloxacillin } \\
(0.8 \%) \text {, ertapenem }(1.6 \%) \text {, imipenem }(10.3 \%) \text {, meropenem }(5.6 \%) \text {, penicillin }(0.8 \%) \text {, } \\
\text { cefoperazone-sulbactam }(2.4 \%) \text {, piperacillin-tazobactam }(8.7 \%) \text {, ampicillin-sulbactam }(2.4 \%)\end{array}$ & 56.3 \\
\hline Glycopeptides vancomycin (10.3\%) & 10.3 \\
\hline $\begin{array}{l}\text { Fluoroquinolones ciprofloxacin }(4.8 \%) \text {, levofloxacin }(0.8 \%) \text {, moxifloxacin }(1.6 \%) \text {, norfloxacin } \\
(0.8 \%)\end{array}$ & 7.9 \\
\hline Aminoglycosides amikacin (4\%), netilmicin (0.8\%) & 4.8 \\
\hline Metronidazole metronidazole (4.8\%) & 4.8 \\
\hline Lincosamides clindamycin (4.8\%) & 4.8 \\
\hline $\begin{array}{l}\text { Others amphotericin B }(3.2 \%) \text {, colistin }(2.4 \%) \text {, fluconazole }(0.8 \%) \text {, fosfomycin }(0.8 \%) \text {, } \\
\text { ganciclovir }(0.8 \%) \text {, itraconazole }(0.8 \%) \text {, ivermectin }(0.8 \%) \text {, roxithromycin }(1.6 \%)\end{array}$ & 11.1 \\
\hline
\end{tabular}

doi:10.1371/journal.pone.0127026.t001 
Table 2. Susceptibility to various antibiotics of $C$. difficile isolates in this study $(\mu \mathrm{g} / \mathrm{mL})$.

\begin{tabular}{|c|c|c|}
\hline Antibiotic & MIC (number of isolates) & $\begin{array}{l}\% \\
\text { Susceptible* }\end{array}$ \\
\hline Metronidazole & $\begin{array}{l}<0.016(9), 0.016(1), 0.023(2), 0.032(5), 0.047(5), \underline{0.064}(12), 0.094 \\
(7), 0.125(9), 0.19(2), 0.5(1)\end{array}$ & $100 \%$ \\
\hline Vancomycin & $\begin{array}{l}0.016(2), 0.023(1), 0.038(1), 0.047(2), 0.064(1), 0.094(1), 0.125(2), \\
0.25(7), 0.38(9), \underline{0.5}(18), 0.75(7), 1.5(1), 3(1)\end{array}$ & $98.2 \%$ \\
\hline Ciprofloxacin & $\underline{\mathbf{3 2}}^{* *}(53)$ & NA \\
\hline Moxifloxacin & $\begin{array}{l}0.032 \text { (1), } 0.25 \text { (1), } 0.38 \text { (5), } 0.5(4), 0.75 \text { (10), } 1 \text { (5), } 1.5 \text { (3), } 24 \text { (1), >32 } \\
(23)\end{array}$ & $54.8 \%$ \\
\hline Linezolid & $\begin{array}{l}0.023(3), 0.032(3), 0.047(3), 0.064(1), 0.094(4), 0.125(5), 0.19(8) \\
0.25(8), 0.38(12), 0.5(3), 0.75(1), 1(1), 2(1)\end{array}$ & NA \\
\hline Tigecycline & $\leq 0.016$ (34), $0.016(7), 0.023$ (9), $0.032(2), 0.064$ (1) & $100 \%$ \\
\hline Daptomycin & $\begin{array}{l}0.023(4), 0.032(4), 0.047(5), 0.064(2), 0.094(4), 0.125(4), \underline{0.19}(7) \\
0.25(5), 0.38(7), 0.5(5), 0.75(5), 1(1)\end{array}$ & $100 \%$ \\
\hline \multicolumn{3}{|c|}{$\begin{array}{l}{ }^{*} \text { Based on EUCAST epidemiological cutoff values (metronidazole } \leq 2 \mu \mathrm{g} / \mathrm{mL} \text {, vancomycin } \leq 2 \mu \mathrm{g} / \mathrm{mL} \\
\text { moxifloxacin } \leq 4 \mu \mathrm{g} / \mathrm{mL} \text {, tigecycline } \leq 0.25 \mu \mathrm{g} / \mathrm{mL} \text {, daptomycin } \leq 4 \mu \mathrm{g} / \mathrm{mL} \text { ) updated } 14 \text { Jan } 2014 \text { (http } \\
\text { www.eucast.org) [20]. } \\
{ }^{*} \mathrm{MIC}_{50} \text { and } \mathrm{MIC}_{90} \text { of ciprofloxacin are identical. Underlined bold numbers are } \mathrm{MIC}_{50} \text {, italicized bold } \\
\text { numbers are } \mathrm{MIC}_{90} . \mathrm{NA}=\text { not applicable. }\end{array}$} \\
\hline
\end{tabular}

doi:10.1371/journal.pone.0127026.t002

\section{PCR ribotyping}

Thirteen RTs were recognized. UK 017 (41.5\%) and UK 014/020 (20.8\%) were the two most common PCR RTs (Fig 1).

\section{MLST}

Thirty ATs and 11 STs were recognized. Among the Thai C. difficile isolates, ST45 and ST33 were the most common (45.3\%) and second most common (24.5\%), respectively. Interestingly, all but one A-B+ strain (22 of 23) belonged to ST45. Additionally, one novel AT of the groEL gene (AT18) and five novel STs (ST66, ST67, ST68, ST69 and ST70) were also found. AT18 and the five novel ST sequences have been submitted to the $C$. difficile MLST database at the Pasteur Institute. The sequences of all the ATs have also been submitted to GenBank under the following accession numbers: KJ130417-446 (S3 Table).

\section{PCR ribotyping, PFGE and MLST method correlations}

Overall, high concordance was observed between the ribotyping and two MLST methods, while PFGE categorized strains differently. For example, most strains of ST14, ST33, ST41 and ST45 correlates with the ribotype QX001 (100\%), UK014/020 (84.6\%), UK046 (83.3\%) and UK017 (91.7\%) with Lamda value of 0.87 (p-value $<0.001$ ). (Fig 1). However, RT UK 014/020 (ST33) correlated with PFGE types 1, 3 and 4 (Fig 1).

\section{Discussion}

The risk factors associated with development of CDI include exposure to antibiotics, advanced age, hospitalization and residency in a long-term care facility [1]. Similar risk factors are also relevant in Thailand where most patients with CDI were of advanced age (mean age 64 years old), had been exposed to antibiotics and were long-term in-patients (96.2\%; mean in-patient duration 62.4 days, data not shown). Analysis of patient medical records showed that beta- 


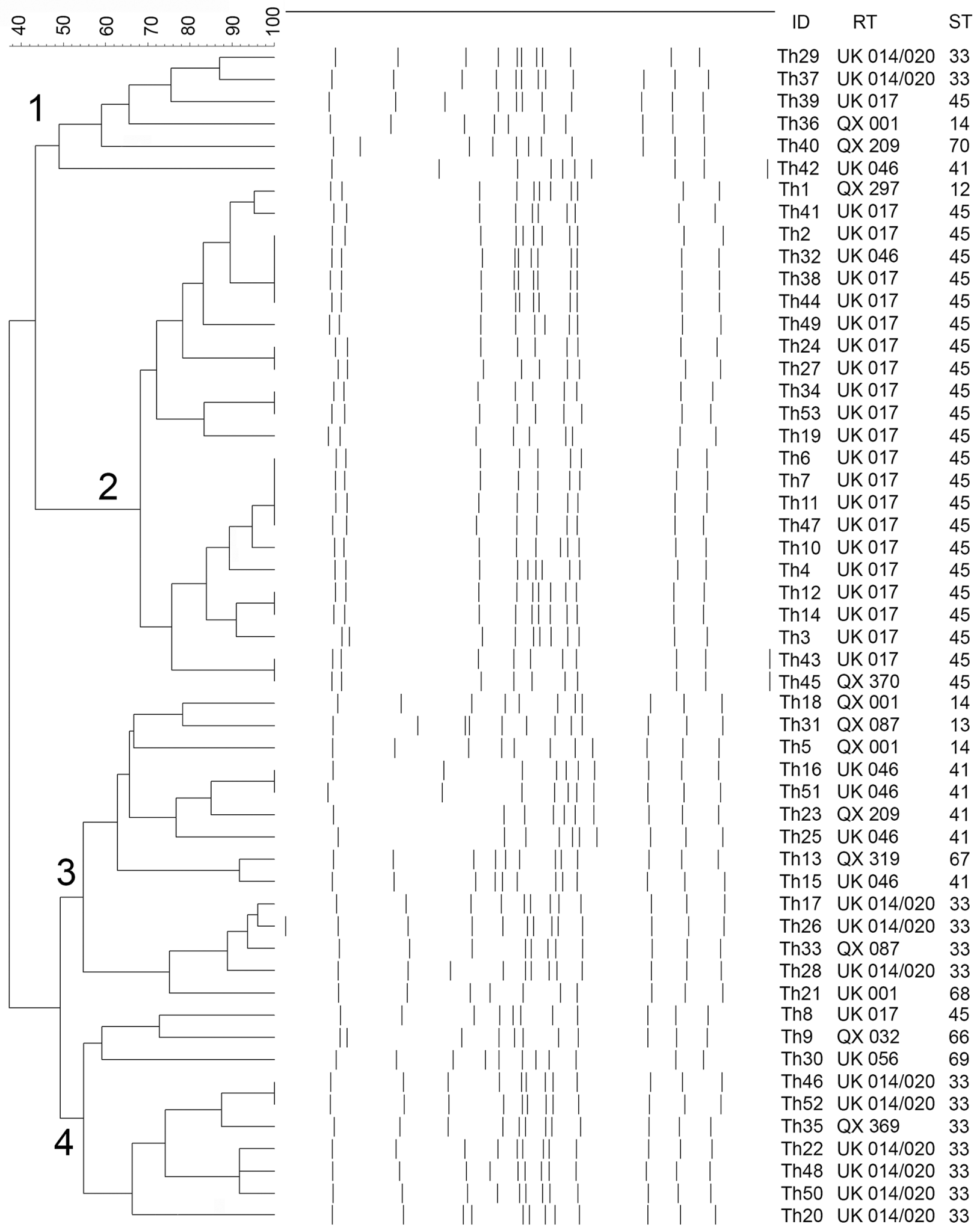

Fig 1. Dendrogram showing PFGE types and their correlations with RTs and STs. Numbers on the branches represent PFGE types. ID = strain identity; $\mathrm{RT}=$ ribotype; ST = sequence type. The scale represents the percentage level of similarity.

doi:10.1371/journal.pone.0127026.g001 
lactams were the most commonly administered antibiotics prior to the acquisition of CDI. This finding is consistent with the scientific literature, whereby antibiotics, including beta-lactams, lincosamides, and fluoroquinolones were frequently associated with CDI [26].

Among the 53 cases, two were young children aged 1 and 6 years old. Studies have found that $C$. difficile strains isolated from children with CDI are often acquired in the community in the absence of previous antibiotic exposure [27]. Although detailed medical records were unavailable, the low MICs of moxifloxacin $(0.5$ and $0.38 \mu \mathrm{g} / \mathrm{mL})$ for the strains isolated from the two children indicate that their infections were acquired outside healthcare settings, particularly as the MICs were well-below the EUCAST epidemiological cut-off value $(\leq 4 \mu \mathrm{g} / \mathrm{mL})$ [28]. More research involving Thai pediatric cohorts is required before further conclusions can be drawn.

As metronidazole and vancomycin are the drugs of choice for CDI treatment [29], their MICs were determined. Although oral vancomycin and fidaxomicin are the only FDA-approved treatments for CDI, metronidazole has also been found to be effective at treating mild to moderate CDIs [13]. Symptom relief was observed in up to $20 \%$ of the cases within the first $48 \mathrm{~h}$ of following these standard treatments [30]. In Thailand, comparable results were observed for oral metronidazole (72.41\%), while vancomycin was shown to be an effective treatment for CDI. Fluoroquinolone treatment is known to be a risk factor for the development of CDI [26]. As such, C. difficile isolates were tested for ciprofloxacin and moxifloxacin resistance. Finally, the MICs of various other antibiotics including linezolid, tigecycline and daptomycin against $C$. difficile were also investigated to determine their suitability as alternative treatments for CDI $[14,30]$. As anticipated, most $C$. difficile strains exhibited high MICs when tested against fluoroquinolones, particularly ciprofloxacin, while low MICs were observed with the new antibiotics, suggesting their potential as new therapeutic options for CDI.

Because CDT was previously found in $4-12 \%$ of toxigenic C. difficile samples [5], the isolates were screened for the presence of $c d t A$ and $c d t B$. Despite CDT-producers increasingly being reported as the causative agents of CDI, our results suggest that this has not occurred in Thailand. Infection with CDT-positive C. difficile is associated with higher mortality and recurrence rates [9]. This is in agreement with our findings that the CDT-negative C. difficile isolated from Thai patients exhibited low virulence and no resistance against standard drug treatments. Moreover, as reported previously [18], the A-B+ variant isolates mostly belonged to the same genotype (UK 017 and ST45). This emphasizes the high correlation observed between the standard genotyping methods and the toxin genotype.

As anticipated from the treatment outcomes, the isolates had high susceptibility levels against metronidazole and vancomycin, which are the first-line drugs for CDI treatment. Similarly, no resistance was observed against the alternative drugs, tigecycline and daptomycin. For linezolid, although an epidemiological cut-off value has not been established, all isolates showed promising MICs of $\leq 2 \mu \mathrm{g} / \mathrm{mL}$, which is a clinical breakpoint for streptococci [20], and lower than those isolated from the United States where strains with MIC as high as $8 \mu \mathrm{g} / \mathrm{mL}$ were isolated from both animals [31] and humans [32]. Additionally, the high MIC values of the isolates against fluoroquinolones was as expected, given the existing scientific literature. Overall, the antibiotic susceptibility of Thai $C$. difficile isolates is in line with surveys from Sweden [14], Taiwan [19] and Canada [33], where high susceptibility to metronidazole and vancomycin, and low susceptibility to fluoroquinolones were reported. However, as the clinical breakpoints for these agents have not been established for C. difficile, interpretation of the MIC values may produce misleading results, and their clinical applications are limited.

Finally, because MLST and ribotyping methods typically exhibit high discriminatory power and are reliable and reproducible, they were used in this study. High overall agreement was observed between the two techniques, which further emphasized their reliability and 
reproducibility. Six of the STs (ST12, ST13, ST14, ST33, ST41 and ST45) among the isolates have also been reported in Europe, North America and Asia [11,34]. The two most common STs in our study (ST45 and ST33) were also among the most common STs in the MLST database containing 539 isolates [34]. Similarly, the rare STs in our study (ST12, ST13 and ST14) were also rare in this database. Interestingly, the hypervirulent PCR RT UK 027 (ST3) [35] was not observed. The absence of the latter and other CDT-producers might have contributed to the low recurrence and mortality rates observed in Thailand thus far [17]. However, to be able to draw this conclusion, further sampling of C. difficile from other area in Thailand is warranted.

\section{Supporting Information}

S1 Table. Primers used in this study.

(DOCX)

S2 Table. Patient demographic data.

(DOCX)

S3 Table. GenBank accession numbers for the sequences of the MLST alleles.

(DOCX)

\section{Acknowledgments}

We thank the Department of Microbiology and Division of Medical Records of the Faculty of Medicine Siriraj Hospital, Mahidol University, for providing study isolates as well as microbiological and patient data. We also appreciate Dr. Thoetchai Peeraphatdit and Mr.Chaitat Laesripa for their technical assistance.

\section{Author Contributions}

Conceived and designed the experiments: PN TVR PK. Performed the experiments: PN SS PPu PPi BE PK. Analyzed the data: PN TVR PK. Contributed reagents/materials/analysis tools: PN TVR PK. Wrote the paper: PN PPu TVR PK.

\section{References}

1. Cecil JA (2012) Clostridium difficile: Changing Epidemiology, Treatment and Infection Prevention Measures. Curr Infect Dis Rep 14: 612-619. doi: 10.1007/s11908-012-0298-9 PMID: 23054932

2. Rupnik M, Grabnar M, Geric B (2003) Binary toxin producing Clostridium difficile strains. Anaerobe 9: 289-294. PMID: 16887714

3. Chia JH, Lai HC, Su LH, Kuo AJ, Wu TL (2013) Molecular epidemiology of Clostridium difficile at a medical center in Taiwan: persistence of genetically clustering of $A(-) B(+)$ isolates and increase of $A(+)$ $\mathrm{B}(+)$ isolates. PLoS One 8: e75471. doi: 10.1371/journal.pone.0075471 PMID: 24116048

4. Rupnik M, Kato N, Grabnar M, Kato H (2003) New types of toxin A-negative, toxin B-positive strains among Clostridium difficile isolates from Asia. J Clin Microbiol 41: 1118-1125. PMID: 12624039

5. Gulke I, Pfeifer G, Liese J, Fritz M, Hofmann F, Aktories K, et al. (2001) Characterization of the enzymatic component of the ADP-ribosyltransferase toxin CDTa from Clostridium difficile. Infect Immun 69: 6004-6011. PMID: 11553537

6. Popoff MR, Rubin EJ, Gill DM, Boquet P (1988) Actin-specific ADP-ribosyltransferase produced by a Clostridium difficile strain. Infect Immun 56: 2299-2306. PMID: 3137166

7. Stubbs S, Rupnik M, Gibert M, Brazier J, Duerden B, Popoff M (2000) Production of actin-specific ADPribosyltransferase (binary toxin) by strains of Clostridium difficile. FEMS Microbiol Lett 186: 307-312. PMID: 10802189 
8. Geric B, Johnson S, Gerding DN, Grabnar M, Rupnik M (2003) Frequency of binary toxin genes among Clostridium difficile strains that do not produce large clostridial toxins. J Clin Microbiol 41:5227-5232. PMID: 14605169

9. Stewart DB, Berg A, Hegarty J (2013) Predicting recurrence of $C$. difficile colitis using bacterial virulence factors: binary toxin is the key. J Gastrointest Surg 17: 118-124; discussion p 124-115. doi: 10. 1007/s11605-012-2056-6 PMID: 23086451

10. Kuijper EJ, van den Berg RJ, Brazier JS (2009) Comparison of molecular typing methods applied to Clostridium difficile. Methods Mol Biol 551: 159-171. doi: 10.1007/978-1-60327-999-4_13 PMID: 19521874

11. Lemee L, Pons JL (2010) Multilocus sequence typing for Clostridium difficile. Methods Mol Biol 646: 77-90. doi: 10.1007/978-1-60327-365-7_6 PMID: 20597004

12. Griffiths D, Fawley W, Kachrimanidou M, Bowden R, Crook DW, Fung R, et al. (2010) Multilocus sequence typing of Clostridium difficile. J Clin Microbiol 48: 770-778. doi: 10.1128/JCM.01796-09 PMID: 20042623

13. Gouliouris T, Brown NM, Aliyu SH (2011) Prevention and treatment of Clostridium difficile infection. Clin Med 11: 75-79. PMID: 21404793

14. Noren T, Alriksson I, Akerlund T, Burman LG, Unemo M (2010) In vitro susceptibility to 17 antimicrobials of clinical Clostridium difficile isolates collected in 1993-2007 in Sweden. Clin Microbiol Infect 16 : 1104-1110. doi: 10.1111/j.1469-0691.2009.03048.x PMID: 19732094

15. Wongwanich S, Ramsiri S, Vanasin B, Khowsaphit $P$, Tantipatayangkul $P$, Phan-urai $R$ (1990) Clostridium difficile associated disease in Thailand. Southeast Asian J Trop Med Public Health 21: 367-372. PMID: 2075479

16. Pupaibool J, Khantipong M, Suankratay C (2008) A study of Clostridium difficile-associated disease at King Chulalongkorn Memorial Hospital, Thailand. J Med Assoc Thai 91: 37-43. PMID: 18386542

17. Thipmontree W, Kiratisin $P$, Manatsathit S, Thamlikitkul V (2011) Epidemiology of suspected Clostridium difficile-associated hospital-acquired diarrhea in hospitalized patients at Siriraj Hospital. J Med Assoc Thai 94 Suppl 1: S207-216. PMID: 21721449

18. Yan Q, Zhang J, Chen C, Zhou H, Du P, Cui Z, et al. (2013) Multilocus sequence typing (MLST) analysis of 104 Clostridium difficile strains isolated from China. Epidemiol Infect 141: 195-199. doi: 10 1017/S0950268812000453 PMID: 22475233

19. Liao CH, Ko WC, Lu JJ, Hsueh PR (2012) Characterizations of clinical isolates of clostridium difficile by toxin genotypes and by susceptibility to 12 antimicrobial agents, including fidaxomicin (OPT-80) and rifaximin: a multicenter study in Taiwan. Antimicrob Agents Chemother 56: 3943-3949. doi: 10.1128/ AAC.00191-12 PMID: 22508299

20. EUCAST (2013) Breakpoint tables for interpretation of MICs and zone diameters (Version 3.1). The European Committee on Antimicrobial Susceptibility Testing.

21. Persson S, Torpdahl M, Olsen KE (2008) New multiplex PCR method for the detection of Clostridium difficile toxin $A(\operatorname{tcd} A)$ and toxin $B(t c d B)$ and the binary toxin $(\operatorname{cdt} A / c d t B)$ genes applied to a Danish strain collection. Clin Microbiol Infect 14: 1057-1064. doi: 10.1111/j.1469-0691.2008.02092.x PMID: 19040478

22. Kato N, Ou CY, Kato H, Bartley SL, Brown VK, Dowell VR Jr., et al. (1991) Identification of toxigenic Clostridium difficile by the polymerase chain reaction. J Clin Microbiol 29: 33-37. PMID: 1993763

23. Stubbs SL, Brazier JS, O'Neill GL, Duerden BI (1999) PCR targeted to the 16S-23S rRNA gene intergenic spacer region of Clostridium difficile and construction of a library consisting of 116 different PCR ribotypes. J Clin Microbiol 37: 461-463. PMID: 9889244

24. Fawley WN, Parnell P, Verity P, Freeman J, Wilcox MH (2005) Molecular epidemiology of endemic Clostridium difficile infection and the significance of subtypes of the United Kingdom epidemic strain (PCR ribotype 1). J Clin Microbiol 43: 2685-2696. PMID: 15956384

25. Hall T (1999) BioEdit: a user-friendly biological sequence alignment editor and analysis program for Windows 95/98/NT. Nucl Acids Symp Ser 41: 95-98.

26. Bouza E, Burillo A, Munoz P (2006) Antimicrobial therapy of Clostridium difficile-associated diarrhea. Med Clin North Am 90: 1141-1163. PMID: 17116441

27. Pant C, Deshpande A, Altaf MA, Minocha A, Sferra TJ (2013) Clostridium difficile infection in children: a comprehensive review. Curr Med Res Opin.

28. Erikstrup LT, Danielsen TK, Hall V, Olsen KE, Kristensen B, Kahlmeter G, et al. (2012) Antimicrobial susceptibility testing of Clostridium difficile using EUCAST epidemiological cut-off values and disk diffusion correlates. Clin Microbiol Infect 18: E266-272. doi: 10.1111/j.1469-0691.2012.03907.x PMID: 22672504 
29. Drekonja DM, Butler M, MacDonald R, Bliss D, Filice GA, Rector TS, et al. (2011) Comparative effectiveness of Clostridium difficile treatments: a systematic review. Ann Intern Med 155: 839-847. doi: 10 7326/0003-4819-155-12-201112200-00007 PMID: 22184691

30. Higa JT, Kelly CP (2013) New Drugs and Strategies for Management of Clostridium difficile Colitis. J Intensive Care Med.

31. Rodriguez-Palacios A, Koohmaraie M, LeJeune JT (2011) Prevalence, enumeration, and antimicrobial agent resistance of Clostridium difficile in cattle at harvest in the United States. J Food Prot 74: 16181624. doi: 10.4315/0362-028X.JFP-11-141 PMID: 22004807

32. Wise R, Andrews JM, Boswell FJ, Ashby JP (1998) The in-vitro activity of linezolid (U-100766) and tentative breakpoints. J Antimicrob Chemother 42: 721-728. PMID: 10052894

33. Bourgault AM, Lamothe F, Loo VG, Poirier L, Group C- CS (2006) In vitro susceptibility of Clostridium difficile clinical isolates from a multi-institutional outbreak in Southern Quebec, Canada. Antimicrob Agents Chemother 50: 3473-3475. PMID: 17005836

34. Lemee L (2011) Clostridium difficile MLST database. Paris, France.

35. Pepin J, Valiquette L, Alary ME, Villemure P, Pelletier A, Forget K, et al. (2004) Clostridium difficile-associated diarrhea in a region of Quebec from 1991 to 2003: a changing pattern of disease severity. CMAJ 171: 466-472. PMID: 15337727 